II Congresso Brasileiro de

Fluidodinâmica Computacional

27 a 29 de Junho de 2018

Rio de Janeiro, RJ

\title{
STREAM FUNCTION-VORTICITY FORMULATION AND HEAT TRANSPORT USING FEM FOR UNSTRUCTURED MESHES AND COMPLEX DOMAINS
}

\author{
L. H. CARNEVALE ${ }^{1}$, G. R. ANJOS ${ }^{2}$, N. MANGIAVACCHI ${ }^{3}$
}

\author{
Universidade do Estado do Rio de Janeiro, Faculdade de Engenharia \\ Email: ${ }^{1}$ Ih.carnevale@gmail.com, ${ }^{2}$ gustavo.anjos@uerj.br, ${ }^{3}$ norberto@uerj.br
}

\begin{abstract}
The stream function-vorticity formulation is a useful alternative to solving the Navier-Stokes equations in two dimensional domain. One of its major difficulties is the lack of boundary conditions for vorticity, and several schemes are proposed to satisfy such a condition. In this paper we propose a finite element scheme for solving the couple problem of the stream functionvorticity formulation using linear triangle elements. A possible application of the method is also demonstrated using the calculated velocity field from the formulation in the heat transport equation to study the temperature distribution in a incompressible single-phase fluid medium. The results obtained were satisfactory when using low to moderate Reynolds number.
\end{abstract}

\section{INTRODUCTION}

In computational fluid dynamics (CFD), solving the incompressible Navier-Stokes equation is of utmost interst in various applications. The stream function-vorticity $(\psi$, $\omega_{z}$ ) formulation is a way to express the Navier-Stokes equation in terms of $\psi$ and $\omega_{z}$ instead of the primitives pressure and velocity (Peeters et al., 1987). The advantage of using the stream function-vorticity formulation is that the pressure does not appear in the equation and, thus, we do not need to solve the pressure-velocity problem, therefore the use of linear element is suitable. The formulation has been proved to be of great use when considering a two dimensional domain (Hoffmann e Chiang, 2000) and in this paper it is proposed an algorithm based on (Salih, 2013) to combine the $\left(\psi, \omega_{z}\right)$ formulation results with the heat transport equation.

\section{MATHEMATICAL MODEL}

For a two dimensional analysis using FEM with unstructured meshes, the proposed method for solving the heat transport equation in a fluid system makes use of the stream function-vorticity formulation (Hoffmann e Chiang, 2000) to obtain a more precise description of the velocity field in a single-phase incompressible flow.

The equations used in this method are:

$$
\omega_{z}=\frac{\partial v_{y}}{\partial x}-\frac{\partial v_{x}}{\partial y}
$$




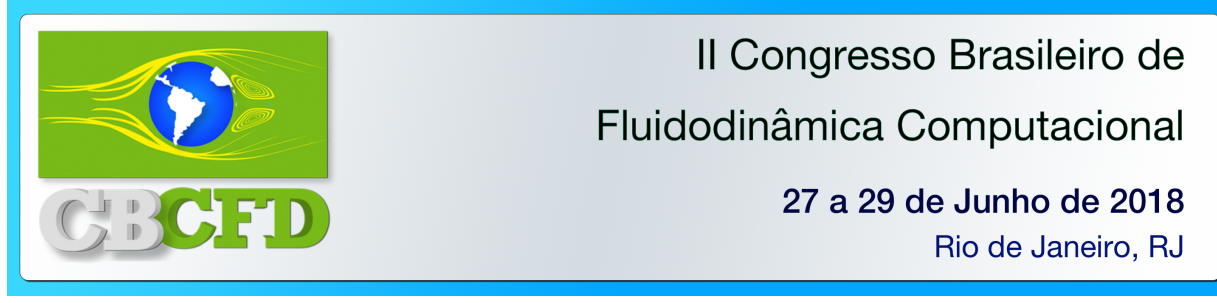

$$
\begin{aligned}
& \frac{\partial \omega_{z}}{\partial t}+\mathbf{v} \cdot \nabla \omega_{z}=\frac{1}{R e} \nabla^{2} \omega_{z} \\
& \frac{\partial^{2} \psi}{\partial x^{2}}+\frac{\partial^{2} \psi}{\partial y^{2}}=\omega_{z} \\
& \frac{\partial \psi}{\partial y}=v_{x}, \quad \frac{\partial \psi}{\partial x}=-v_{y} \\
& \frac{\partial T}{\partial t}+\mathbf{v} \cdot \nabla T=\frac{1}{\operatorname{RePr}} \nabla^{2} T+Q
\end{aligned}
$$

Where $T$ is the scalar field of temperature, the Reynolds number is $R e=\rho u D / \mu$ ( $\rho$ is the density, $u$ is the reference velocity, $\mu$, is the viscosity and $D$ is a characteristic linear dimension), the Prandtl number is $\operatorname{Pr}=\nu / \alpha$ ( $\nu$ is the kinematic viscosity and $\alpha$ is the thermal diffusivity), $\omega_{z}$ is the vorticity field, $x$ and $y$ are the independent spatial variables, $\mathbf{v}=\left(v_{x}, v_{y}\right)$ is the velocity field, $\psi$ is the stream function, $Q$ is the generation of heat, and $t$ is the time variable.

The algorithm developed to solve the stream function-vorticity problem was based on the algorithm proposed on (Salih, 2013). At first an initial v field is initialized, respecting the boundary conditions for velocity, and the initial $\omega_{z}$ is calculated with equation (1). After that, the initial $\psi$ function is obtained by solving equation(3) with Dirichlet boundary conditions for $\psi$.

The next step is to obtain the boundary conditions for the vorticity that are needed to solve eqation (2). Various finite differences schemes can be derived for that, such as in (Peeters et al., 1987), however this schemes need a structured mesh to be implemented. The proposed way to calculate the boundary conditions when using unstructured meshes is to calculate $\omega_{z}$ with equation (1) in each time step as the velocity field changes.

With the initial values for $\mathbf{v}, \omega_{z}$ and $\psi$ and the boundary conditions calculated, the vorticity transport equation (2) is solved for the next time step, than with the new $\omega_{z}$ field, equation (3) is solved for the new $\psi$ field. Afterwards the new velocity field is obtained by equations in (4). This repeats over a defined number of steps.

In the equations (1) and (4) we use the Lumped Mass matrix to reduce the computational cost of inverting the original Mass matrix $(\mathbf{M})$. The Lumped matrix is constructed adding all the elements for each line of $\mathbf{M}$ and placing the results in the main diagonal and its use is valid when the original matrix elements have higher values when closer to its diagonal.

The FEM algorithm was developed for the use of linear triangular elements in the discretization of the spatial domain and for the time it was used a finite differences method with a forward difference approximation and an implicit approach. The linear systems of equations were solved by a direct solver. To build the geometric model and mesh of the different spatial domains an open source software called "GMSH" Geuzaine e Remacle (2009) was used. 


\section{RESULTS}

\subsection{Poiseuille Flow in Heated Plates}

A validation for the temperature equation (5) was constructed based on the analytic solution shown in (Pontes e Mangiavacchi, 2010). After apllying all the coeficient values, the temperature solution can be described by equation (6).

$$
T=\frac{-15}{48} q+\frac{2}{R e(-\partial P / \partial x)} q x+3 q\left(\frac{y^{2}}{2}-\frac{y^{4}}{3}\right)
$$

The top and bottom plates had a heat flux boundary condition of $q=1$ perpendicular to the boundary. For the stream function $\psi=0$ on the bottom plate and $\psi=1$ on the top plate, $R e=10, \operatorname{Pr}=1$, and $(-\partial P / \partial x)=12$. The exact solution for temperature and numerical solutions are shown in Figure 1 and Figure 2 after 60 iterations with $\Delta t=0.1$.

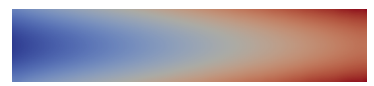

(a) Temperature

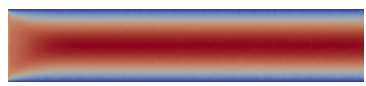

(b) Velocity profile

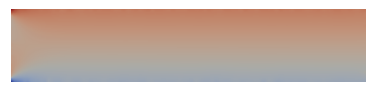

(c) Vorticity

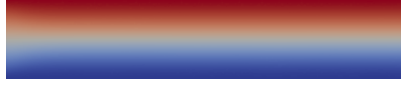

(d) Stream Function

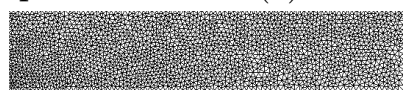

(e) Unstructured Triangular Mesh

Figure 1 - Numerical solutions obtained by the stream function-vorticity FEM code

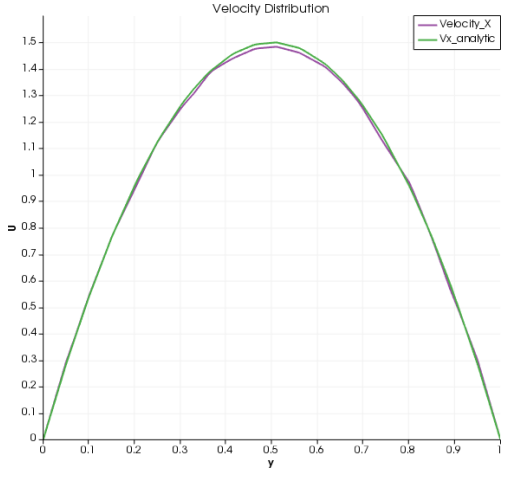

(a)

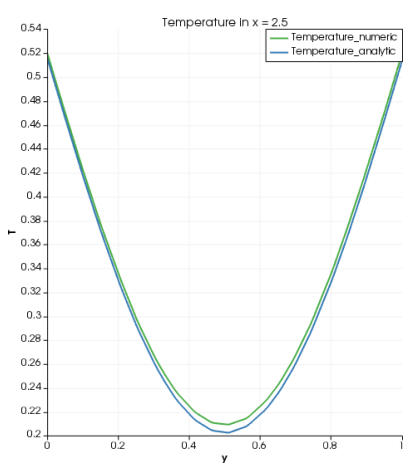

(b)

Figure 2 - Comparison of the numerical soluion obtaind with the stream function-vorticity finite element solver and the exact solution for (a) Velocity comparison; (b) Temperature comparison 


\subsection{Heat Transport Between Non-Smooth Plates}

The results presented in figure Figure 3 are for a similar case as the above, however now the heat flux $q=1$ is only defined on the rectangular shapes along the top and bottom boundaries with the intent to observe how a perturbation on the boundary would affect the solution. Each rectangle has a 0.1 height and 0.5 width. The other boundary conditions and simulation parameters were set as the same for the plane Poiseuille case.

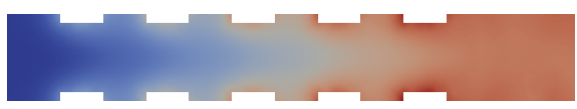

(a) Temperature

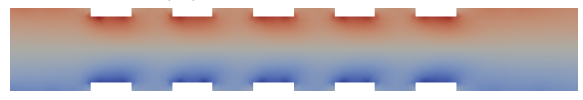

(c) Vorticity

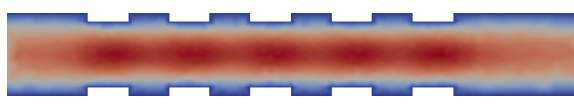

(b) Velocity profile

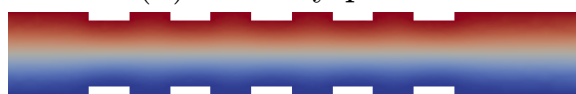

(d) Stream Function

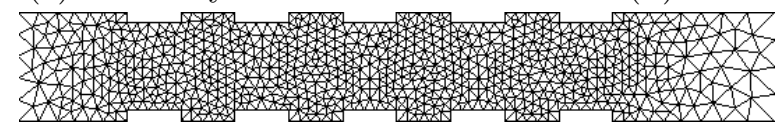

(e) Unstructured Triangular Mesh

Figure 3 - Numerical solutions obtained by the stream function-vorticity FEM code for the complex geometry proposed

\section{CONCLUSION}

The proposed method for solving the stream function-vorticity system using the FEM on two dimensional unstructured meshes has shown good results when compared with the literature on the case of low Reynolds. Moreover, it has also been demonstrated its aplication alongside the temperature distribution equation. The algorithm is in continuous development to acomodate higher $R e$ values, thus simulating more challenging problems.

\section{REFERENCES}

Geuzaine, C.; Remacle, J.-F. Gmsh: a three-dimensional finite element mesh generator with built-in pre- and post-processing facilities. International Journal for Numerical Methods in Engineering, 2009.

Hoffmann, K. A.; Chiang, S. T. Computational Fluid Dynamics, volume 1. Engineering Education Systems, fourth edição, 2000.

Peeters, M. F.; Habashit, W. G. .; Dueck, E. G. . Finite element stream functionvorticity solutions of the incompressible navier-stokes equations. International Journal for Numerical Methods in Fluids, 1987.

Pontes, J.; Mangiavacchi, N. Fenômenos de Transferência, volume 1.1 edição, 2010.

SALIH, A. Streamfunction-vorticity formulation. Indian Institute of Space Science and Technology, Department of Aerospace Engineering, 2013. 